\title{
Cutaneous and Ganglion Sarcoidosis Induced by Polycaprolactone Facial Filler: A New Expression of ASIA Syndrome?
}

\author{
Juan Ortiz-Álvarez ${ }^{1}$, Jose Antonio Lebrón-Martín'르. Lourdes Rodríguez Fernández-Freire ${ }^{1}$, \\ Teresa Zulueta Dorado², José Salvador García Morillo ${ }^{3}$ \\ ${ }^{1}$ UGC de Dermatología, Hospital Universitario Virgen del Rocío, Sevilla, Spain \\ 2 UGC de Anatomía Patológica, Hospital Universitario Virgen del Rocío, Sevilla, Spain \\ ${ }^{3}$ Unidad de Enfermedades Raras y Autoinmunes del Adulto, UGC Medicina Integral, Hospital Universitario Virgen del Rocío, Sevilla, Spain
}

\section{Doi: 10.12890/2021_002652- European Journal of Case Reports in Internal Medicine - @ EFIM 2021}

Received: $18 / 05 / 2021$

Accepted: $20 / 05 / 2021$

Published: 09/06/2021

How to cite this article: Ortiz-Álvarez J, Lebrón-Martín JA, Rodríguez Fernández-Freire L, Zulueta DoradoT, García Morillo JS. Cutaneous and ganglion sarcoidosis induced by polycaprolactone facial filler: a new expression of ASIA syndrome? EJCRIM 2021;8: doi:10.12890/2021_002652.

Conflicts of Interests: The authors declare there are no competing interests.

Patient consent: Informed consent was obtained from the patient for reproduction of the clinical images.

This article is licensed under a Commons Attribution Non-Commercial 4.0 License

\section{ABSTRACT}

Dermal fillers are applied using a minimally invasive technique with a good safety profile. However, they can have side effects. We present the case of a patient who, 2 months after undergoing polycaprolactone (Ellansé ${ }^{\circledR}$ ) injections, developed nodular facial and nodal lesions that were compatible with sarcoidosis on histology. This complication has not been previously described for polycaprolactone and could be the expression of an autoimmune syndrome induced by adjuvants.

\section{LEARNING POINTS}

- Autoimmune/autoinflammatory syndrome induced by adjuvants (ASIA) is a recently devised umbrella term for autoimmune diseases caused by adjuvants.

- Aesthetic procedures, which are increasingly common, may be a cause of ASIA syndrome.

- Polycaprolactone is a bioabsorbable polymer with a safe profile but can have adverse events, including systemic sarcoidosis.

\section{KEYWORDS}

Polycaprolactone filler, sarcoidosis, ASIA syndrome

\section{INTRODUCTION}

Sarcoidosis is a multisystemic granulomatous disease caused by an abnormal immune reaction to antigenic stimuli, with Th1 lymphocytes, interferon gamma and tumour necrosis factor (TNF) playing important roles ${ }^{[1]}$.

Aesthetic procedures, especially those employing minimally invasive techniques which are therefore quick and accessible, are increasing in popularity. However, such procedures can result in adverse effects, and granulomatous and foreign body reactions to injectables are well known ${ }^{[2]}$. Polycaprolactone (PCL) (Ellansé $\left.{ }^{\circledR}\right)$ is a recently developed bioabsorbable medical polymer which has emerged as a durable and safe dermal filler that allows immediate remodelling of facial features and stimulates the production of collagen by the body ${ }^{[3]}$.

We present a case of systemic sarcoidosis triggered by the use of facial fillers containing PCL, a complication not previously described for this material. 


\section{CASE DESCRIPTION}

A 74-year-old woman, with no relevant medical history, was referred to dermatology consultations for evaluation of asymptomatic facial skin lesions with 1 year of evolution. She reported having had an Ellansé ${ }^{\circledR}$ (PCL) implant in a private clinic for cosmetic purposes about 3 months before lesion onset. She had no other associated symptoms, although during follow-up she had complained of myalgia and dizziness. Physical examination revealed four subcutaneous and indurated $2 \times 2 \mathrm{~cm}$ nodules, located bilaterally and symmetrically in the lower part of both nasolabial folds and over both zygomatic arches (Fig. 1). Biopsy results revealed chronic granulomatous inflammation, with multinucleated giant cells affecting the adipose panniculus (Fig. 2). Staining for microorganisms and microbiological studies were negative and the diagnosis of sarcoid granulomas was made by the dermato-pathologist. A haemogram, immunoglobulin tests and chest $\mathrm{x}$-ray did not show any notable findings. Angiotensin-converting enzyme (ACE) was slightly elevated (20 IU/I), as was ESR (30 mm/h). The Mantoux intradermal test and IGRA were negative. Therefore, a head, neck and chest CT scan was performed, which showed large adenopathic conglomerates in all mediastinal compartments, compatible with sarcoidosis, and without alterations in the lung parenchyma.

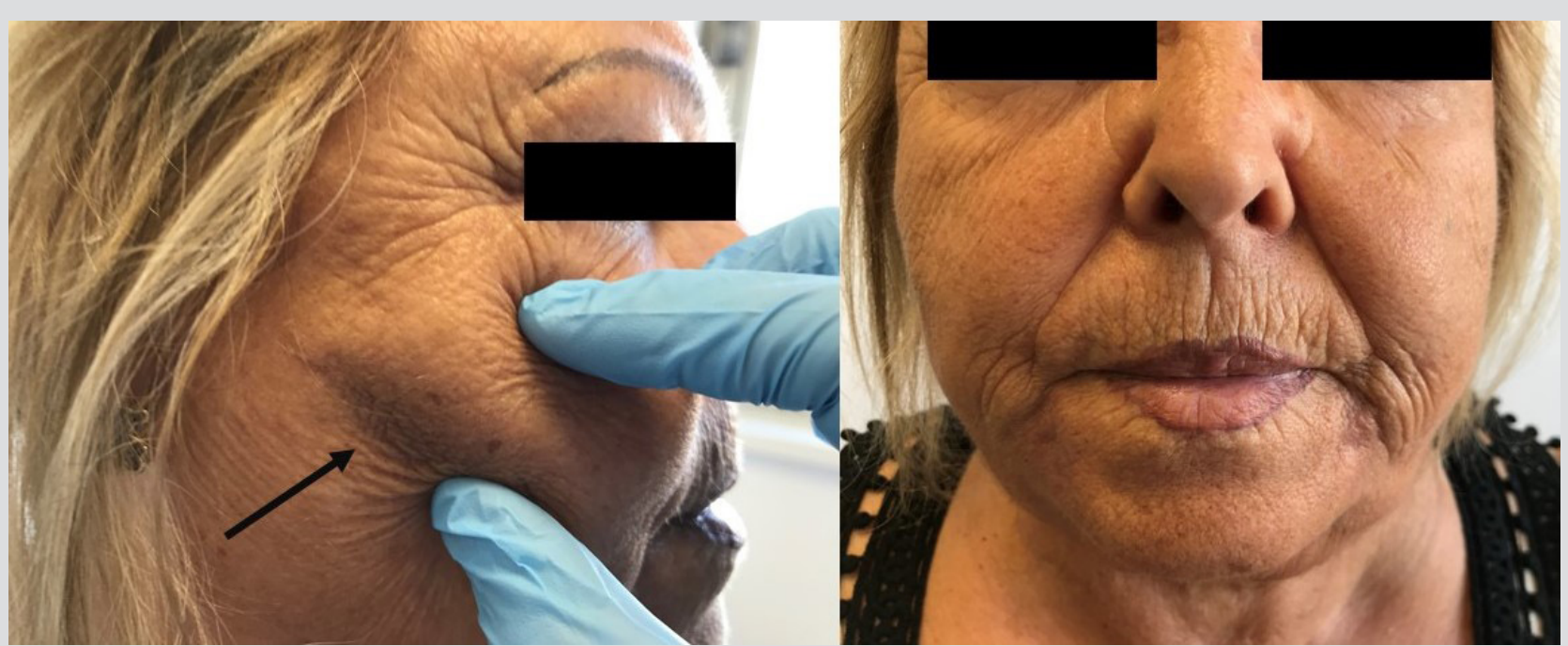

Figure 1. Dermatological findings in facial skin

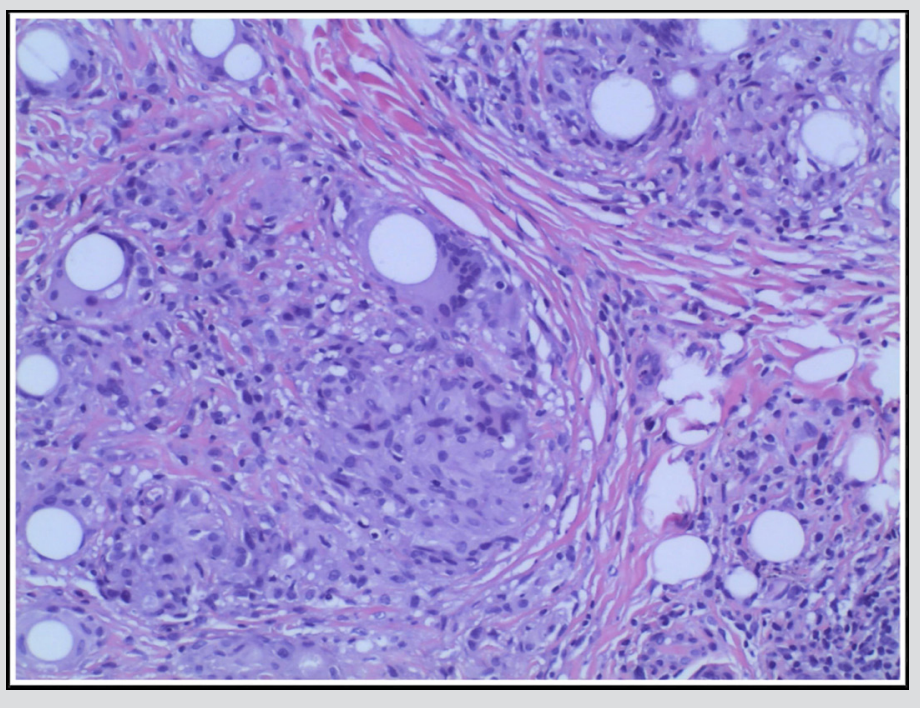

Figure 2. Histological findings of one of the facial lesions

Treatment with prednisone $(0.7 \mathrm{mg} / \mathrm{kg} / \mathrm{day})$ was initiated but discontinued after 3 months due to lack of response. Subsequently, methotrexate (20 mg subcutaneous weekly) was started in combination with prednisone $(0.17 \mathrm{mg} / \mathrm{kg} /$ day) with significant aesthetic and symptom improvement (Fig. 3). 


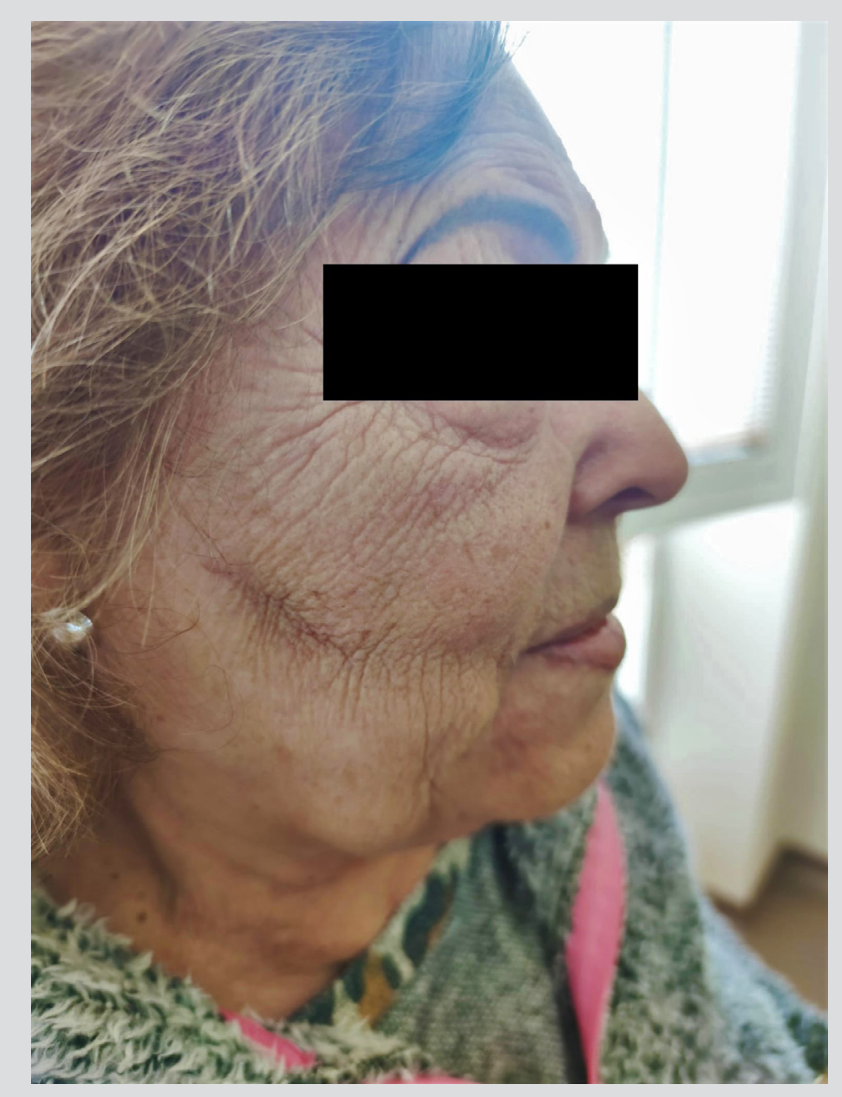

Figure 3. Dermatological findings after methotrexate

\section{DISCUSSION}

The association of sarcoidosis with cosmetic procedures such as tattooing or silicone facial filler injection, is well known ${ }^{[1]}$. In a recent review of 1111 patients treated with PCL injections, an incidence of adverse effects of $4.5 \%$ was observed, consisting of bruises, malar oedema, discoloration or palpable nodules, but no cases of sarcoidosis were seen ${ }^{[3]}$. Biomaterials induce an immune response and macrophage mobilization, and it has been reported that PCL stimulates a standard foreign body response characterized by TH17 cells, IL-17 and fibrosis ${ }^{[4]}$. In 2011, Shoenfeld and Agmon-Levin proposed the acronym ASIA (autoimmune/autoinflammatory syndrome induced by adjuvants) as an umbrella term for a series of autoimmune disorders induced by adjuvants, substances capable of amplifying and maintaining autoimmune responses caused by other antigens ${ }^{[5]}$. Implants and silicone injections have been reported to trigger ASIA syndrome, and it is hypothesized that sarcoidosis could be included in this entity ${ }^{[5]}$.

Our patient developed one of the most common forms of cutaneous sarcoidosis, with primary involvement of subcutaneous tissue and the formation of palpable nodules in the implant area a few months after injection ${ }^{[1]}$. This spatio-temporal relationship suggests the filler acted as an adjuvant and reinforces the suggestion that sarcoidosis should be included in ASIA syndrome. Moreover, apart from HLA determination and surgical removal of the implants (which the patient refused), this case meets the current diagnostic criteria for this entity: exposure to an external stimulus, latency, locoregional and systemic clinical signs, and compatible biopsy findings ${ }^{[5]}$.

\section{CONCLUSION}

We report a previously undescribed complication of PCL injections. In addition, the described side effect could be considered an example of ASIA syndrome, although deeper knowledge of the subject and greater consensus on which entities could benefit from this definition is needed. 


\section{REFERENCES}

Wanat KA, Rosenbach M. Cutaneous sarcoidosis. Clin Chest Med 2015;36(4):685-702. Available from: http://dx.doi.org/10.1016/j.ccm.2015.08.010 Levy L, Emer J. Complications of minimally invasive cosmetic procedures: prevention and management. J Cutan Aesthet Surg 2012;5(2):121-132.

Lin SL, Christen MO. Polycaprolactone-based dermal filler complications: a retrospective study of 1111 treatments. J Cosmet Dermatol $2020 ; 19$ (8):1907-1914.

4. Sommerfeld S, Cherry C, Schwab R, Chung L, Maestas DJ, Laffont P, et al. Interleukin-36y-producing macrophages drive IL-17-mediated fibrosis. Sci Immunol 2019;11(4):eaax4783.

5. Alijotas-Reig J. Human adjuvant-related syndrome or autoimmune/inflammatory syndrome induced by adjuvants. Where have we come from? Where are we going? A proposal for new diagnostic criteria. Lupus 2015;24(10):1012-1018. 